\title{
Hemisection - ray of hope for hopeless tooth in a patient with progressive destruction of periodontium: a rare case report
}

\author{
Simran Parwani ${ }^{1 *}$, Nikita Rathi ${ }^{1}$, Shruti Wankhade ${ }^{1}$ and Shatakshi Bartere ${ }^{2}$ \\ ${ }^{1}$ Department of Periodontology, V.Y.W.S. Dental College and Hospital, India \\ ${ }^{2}$ Department of Conservative Dentistry and Endodontics, V.Y.W.S. Dental College and Hospital, India
}

\begin{abstract}
Hemi-section refers to the sectioning of a molar tooth with the removal of unrestorable and/or periodontally compromised crown and root, which may be affected by periodontal, endodontic or structural failures. It helps in saving a tooth which otherwise would have been deemed for extraction. In this case report of patient with generalised progressive destruction of periodontium, an advanced Endo-Perio lesion with right mandibular first molar was successfully treated by root-canal treatment followed by hemisection and flap surgery. It describes a procedure of hemisection in mandibular molar with periodontally compromised mesial root; restoration of periodontium in the mesial root socket with bone graft and PRF and its subsequent prosthetic restoration. Six month clinical and radiographic follow-ups depicted retention of distal half of tooth structure and its supporting periodontium; in addition to being cost-effective treatment.
\end{abstract}

\section{Introduction}

Hemisection involves removing periodontally compromised root structure and associated coronal structure in a furcation involved tooth. The treatment goal is the conservation of remaining tooth structure and restoration of function [1]. Progressive inflammatory periodontal disease, left untreated, will ultimately result in attachment and tooth loss. Management of periodontally involved molars is a challenge and is limited to dental extraction and replacement with implants [2]. Due to advances in dentistry, a conservative treatment strategy to retain such teeth involves periodontal, prosthodontic, and endodontic assessment; making it possible to maintain a functional dentition for a lifetime [3]. Hemisection procedure is done where only one root of a multirooted tooth is affected and the surviving root is structurally capable of supporting a dowel and core restoration. The present report depicts one such case in a patient with generalized progressive destruction of periodontium.

\section{Case report}

A 36 years old systemically healthy male patient reported with the complaint of bleeding gums with dull aching pain and mobility of right mandibular first molar since last 2-3months. On clinical and radiographic examination, it was found that amount of local irritants was not commensurate with the amount of bone destruction with generalized moderate to severe periodontitis. Right mandibular first molar was sensitive to vertical percussion and exhibited grade I mobility and grade III furcation involvement. On probing the area with periodontal probe there was a $14 \mathrm{~mm}$ deep periodontal pocket around the mesial root and six $\mathrm{mm}$ around distal root of the same tooth. On radiographic examination, severe bone loss $(>50 \%)$ was evident surrounding the mesial root and involving the furcation area. After all clinical, radiographic and blood investigations (haemoglobin percentage, complete blood count, bleeding and clotting times, total and differential leucocyte counts and random blood sugar); diagnosis was made of generalized progressive destruction of periodontium with advanced endo-perio lesion involving mesial root of right mandibular first molar (Figure 1). It was decided that the mesial root along with adjoining crown portion should be hemisected after completion of endodontic therapy of the tooth along with treatment of progressive destruction of periodontium. The treatment plan was elucidated to the patient, prognosis of hemisection against extraction of the tooth discussed and consent obtained.

Initially full mouth disinfection including scaling and root planing was done and antimicrobial therapy (amoxicillin $500 \mathrm{mg}$ TDS and metronidazole $400 \mathrm{mg}$ TDS) was started from the same day for next 14 days. In the meanwhile, access opening was done for 46 and working length of the tooth was determined. Biomechanical preparation was done, and master cone radiograph was taken. The canals were obturated with crown-down method, post-endodontic restoration was performed with composite material (Figure 2a-2d). Patient was evaluated six weeks after full mouth disinfection where it was found that disease had entered inactive stage but there was still presence of moderate to severe periodontal pocket. Under antibiotic coverage open flap debridement and necessary resective/regenerative procedure was done with first, second and third quadrants.

*Correspondence to: Simran Parwani, Professor and Head of Department, Department of Periodontology, V.Y.W.S. Dental College and Hospital, India, E-mail: simpar74@gmail.com

Key words: hemisection, progressive destruction of periodontium, bone loss, furcation, prosthetic rehabilitation

Received: February 18, 2020; Accepted: February 27, 2020; Published: March 03, 2020 
For the treatment of hemisection, before flap reflection crown portion was sectioned into mesial and distal half up to the level of CEJ using high speed air-rotor, hand piece and diamond burs (Figure 3a-3d). Under local anesthesia, full thickness flap was reflected after giving a crevicular incision for the whole quadrant (Figure 3e). All granulation tissue was debrided with Gracey curettes (Hu-Friedy, USA) to expose $1 \mathrm{~mm}$ of alveolar bone. The vertical cut method was used to resect the remaining crown portion along with root. A long shank tapered fissure diamond bur with contra-angled micromotor

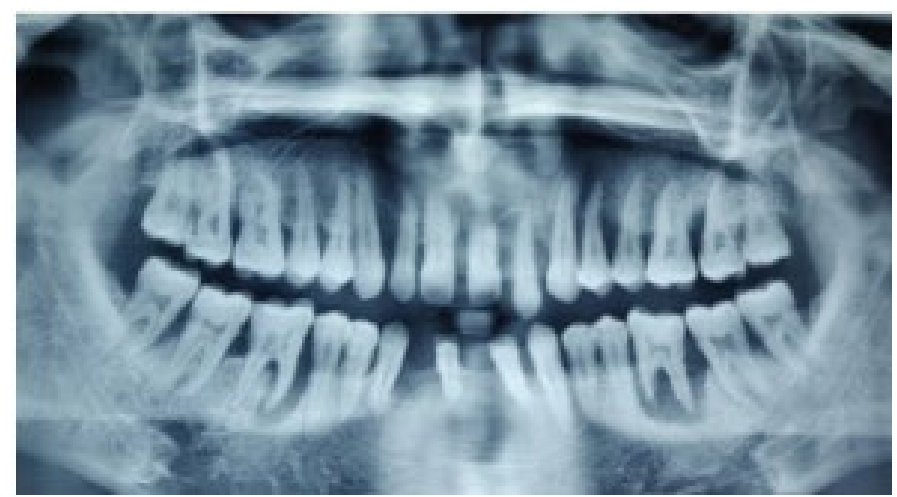

Figure 1. Pre-operative OPG

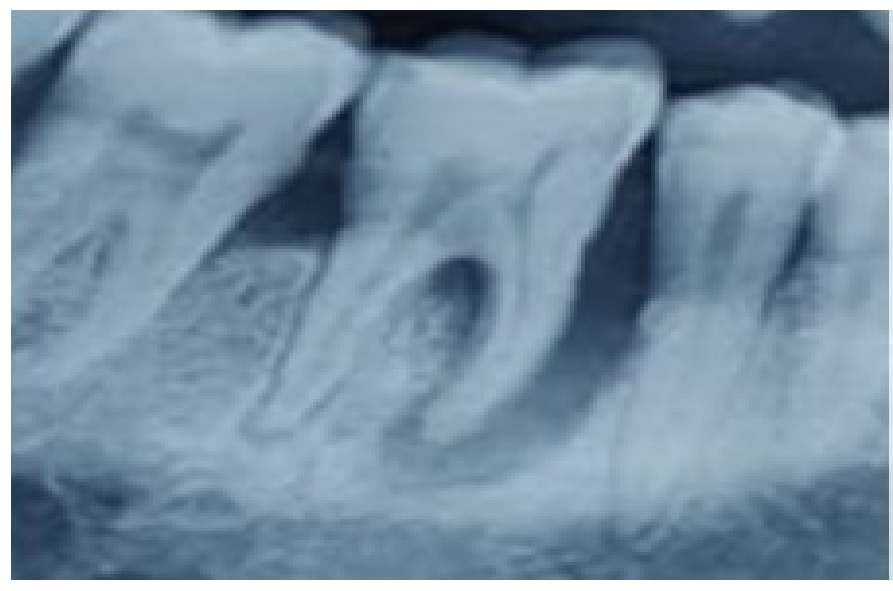

Figure 2a. Pre-operative IOPA

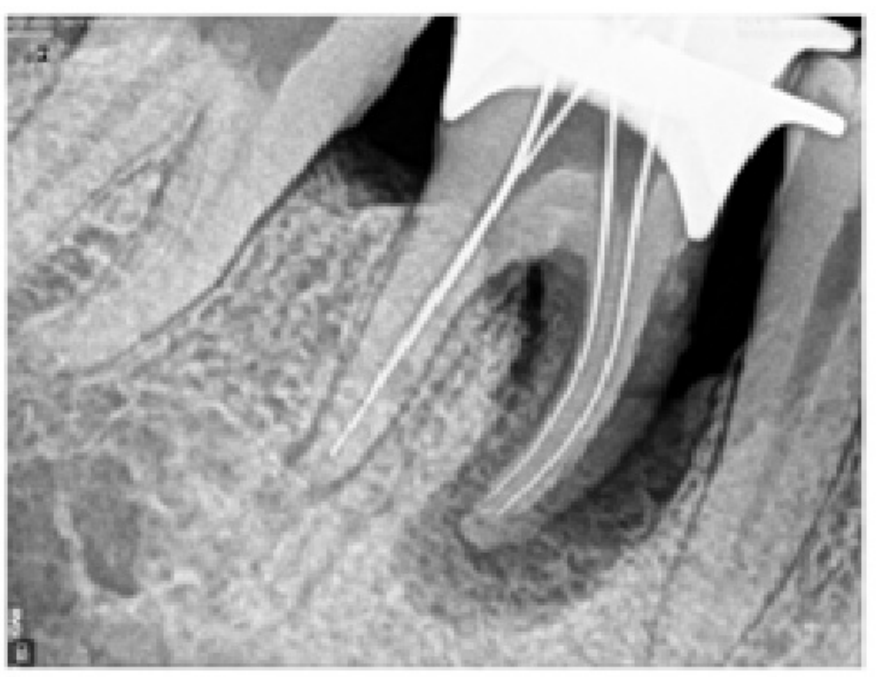

Figure 2b. Working length RVG

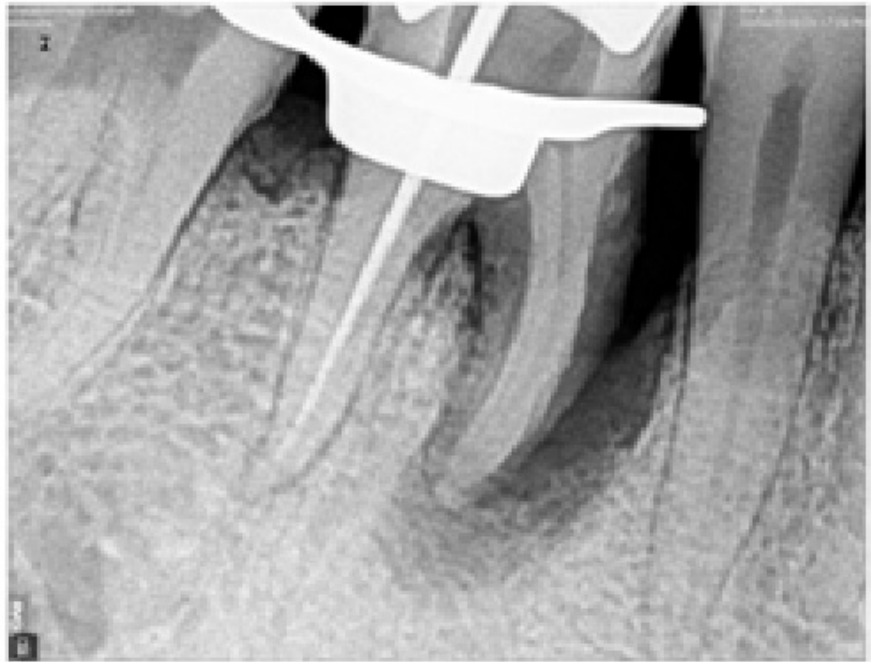

Figure 2c. Master cone RVG

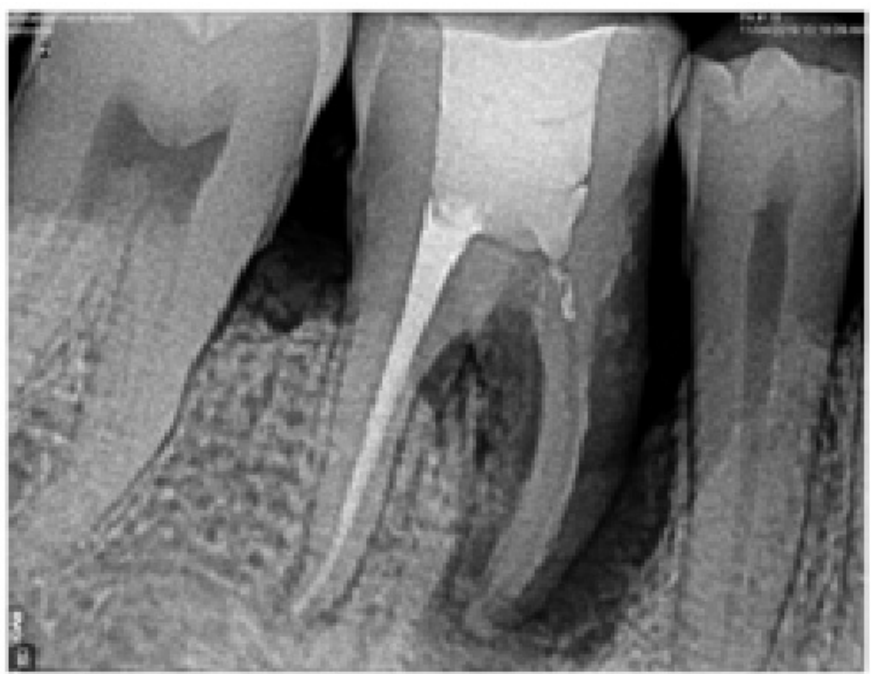

Figure 2d. Post- obturation RVG

handpiece was used to make vertical cut towards the bifurcation area (Figure 3f). A straight probe was passed through the cut to assure separation. The mesial half was elevated and extracted atraumatically, socket was curetted and later irrigated adequately with sterile saline subsequently filed with bone files to reshape irregularities (Figure $3 \mathrm{~g}$ ). The occlusal table was reformed to redirect the forces along the long axis of the distal root (Figure 3h). Radiographs showed the well retained distal root. Presuturing was done and to enhance the osteogenesis and healing in the extraction socket, platelet rich fibrin along with demineralized freeze dried bone allograft (DFDBA) followed by bioresorbable collagen membrane (healiguide), were placed after dipping it in the saline for 2-3 minutes (Figure 3i-3k). Tin foil of appropriate size was placed at the surgical site and flap was replaced, and simple interrupted sutures were placed. The surgical site was covered by periodontal dressing (Coe Pak ${ }^{\mathrm{mm}}$ GC America Inc., Alsip, IL, USA), and postoperative instructions including prescription of antibiotics and analgesics were given to the patient. Sutures were removed 14 days post- operatively. Patient was followed up by regular monthly visits. Six months clinical and radiographic follow-ups showed uneventful healing at surgical site (Figure $4 \mathrm{a}-4 \mathrm{~b}$ ). Patient was then referred to department of Prosthodontics for prosthesis. Prosthodontic treatment 


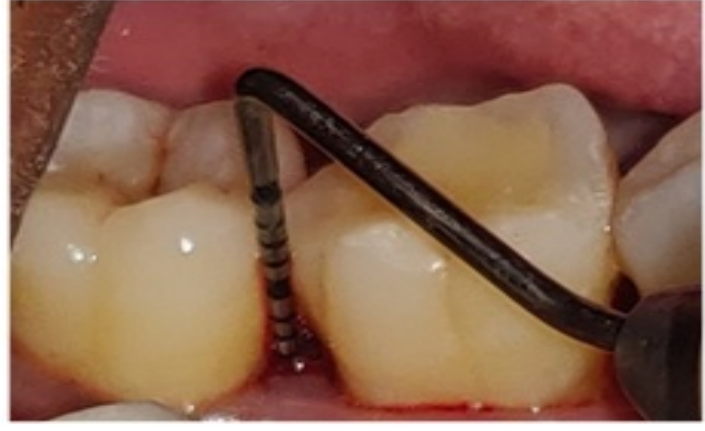

Figure 3a. Probing pocket depth at mesial root

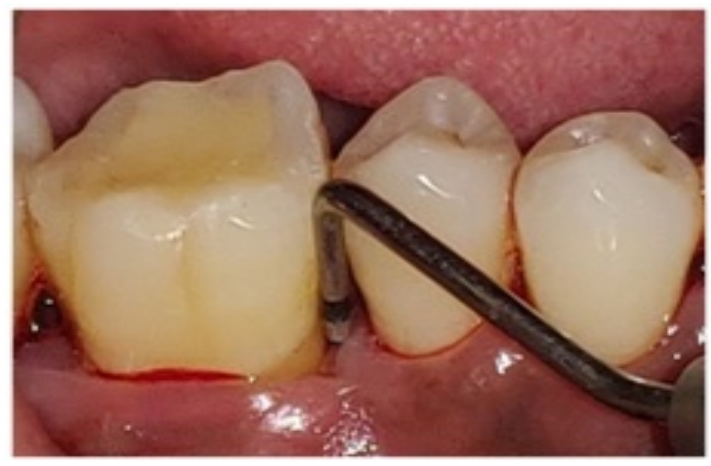

Figure 3b. Probing pocket depth at distal root

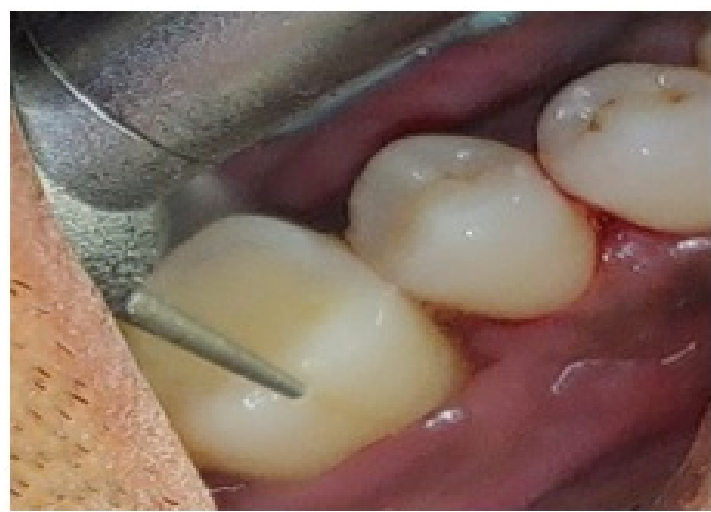

Figure 3c. Crown sectioning done using high speed air-rotor handpiece

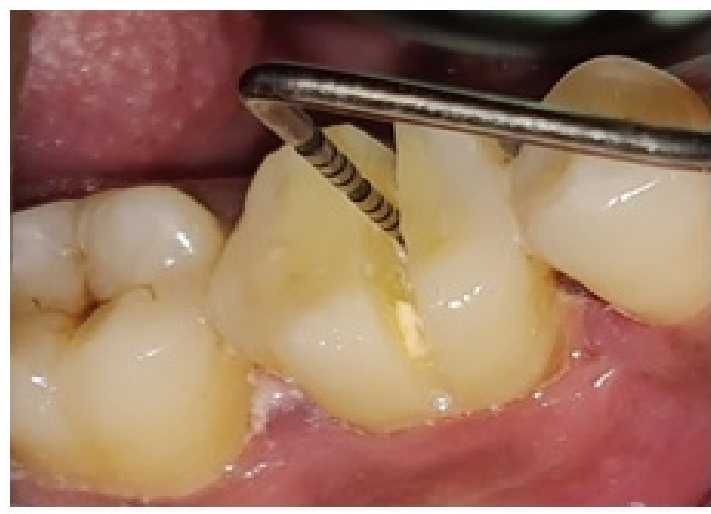

Figure 3d. Confirmation of separation done using probe

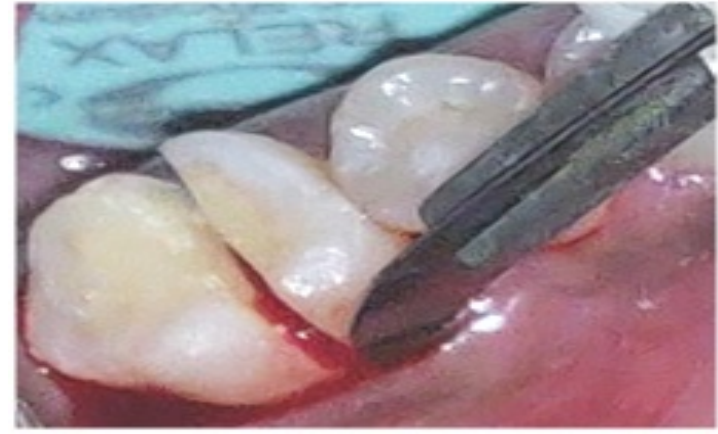

Figure 3e. Sulcular incision and flap reflection

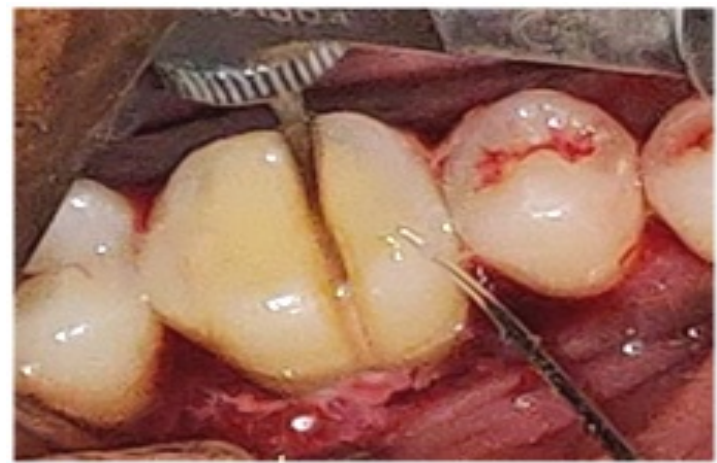

Figure 3f. Separation using micromotor handpiece and diamond bur along with irrigation

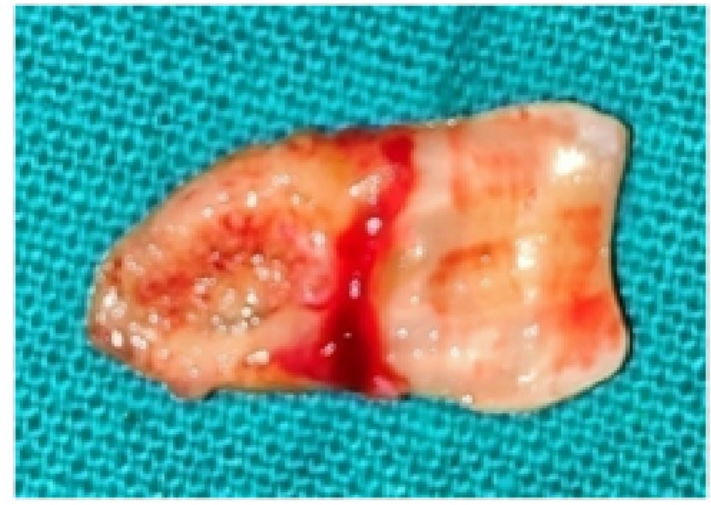

Figure 3g. Extracted hemisectioned crown along with mesial roo

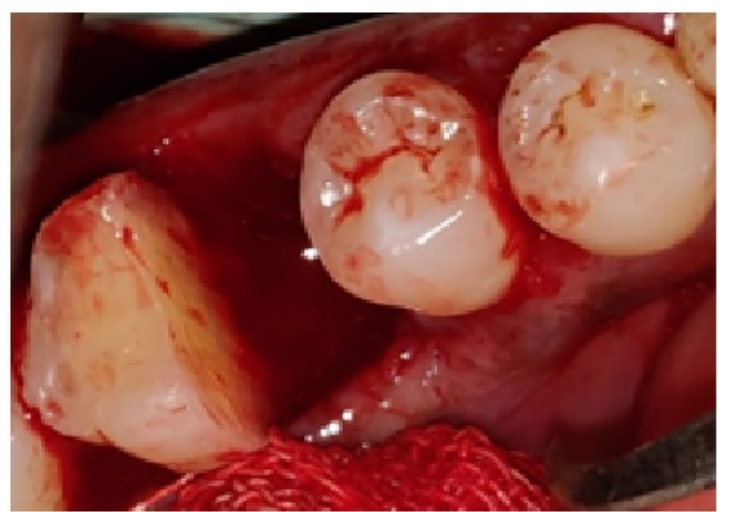

Figure 3h. Occlusal view of socket 


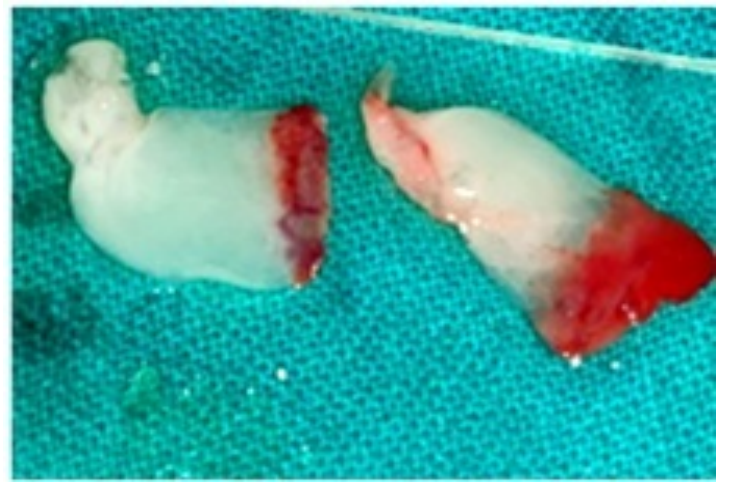

Figure 3i. PRF preparation

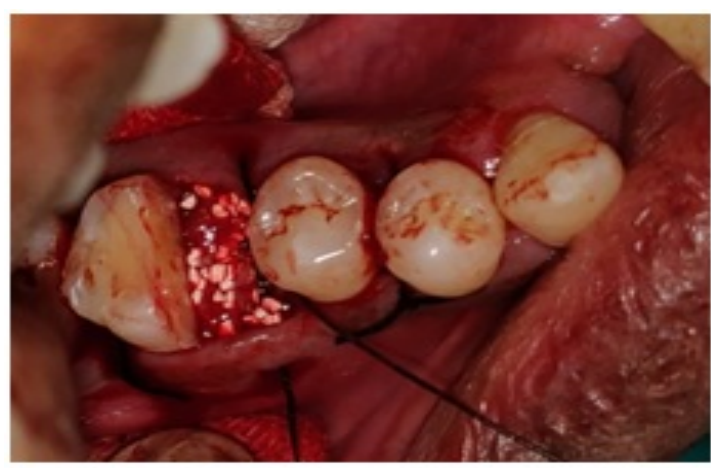

Figure 3j. Bone graft and PRF placement

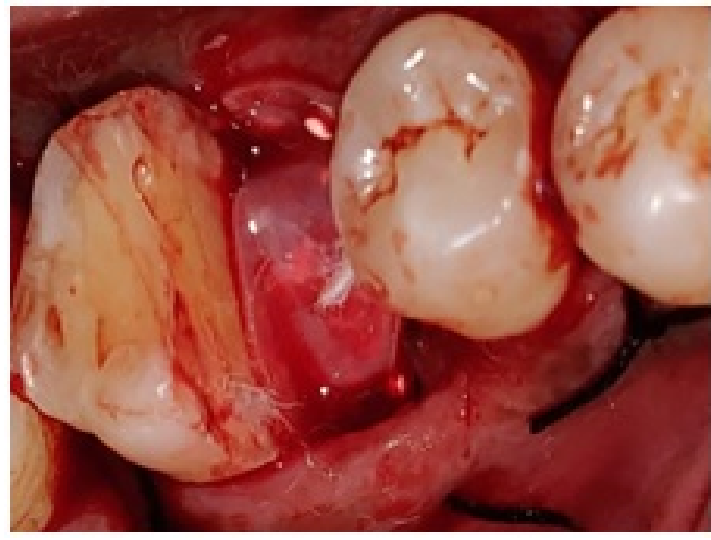

Figure 3k. Bioresorbable Membrane placement

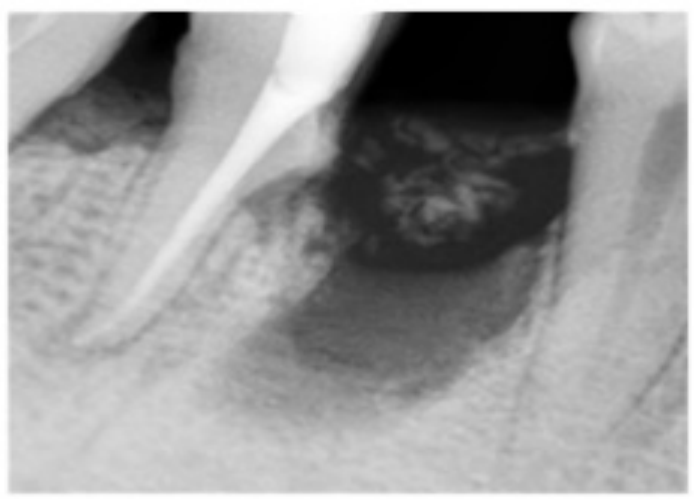

Figure 4a. Immediate post- operative radiograph included taking facebow records, followed by tooth preparation and permanent restoration of teeth (Figure 5a-5d). Post cementation RVG is shown here (Figure 6).

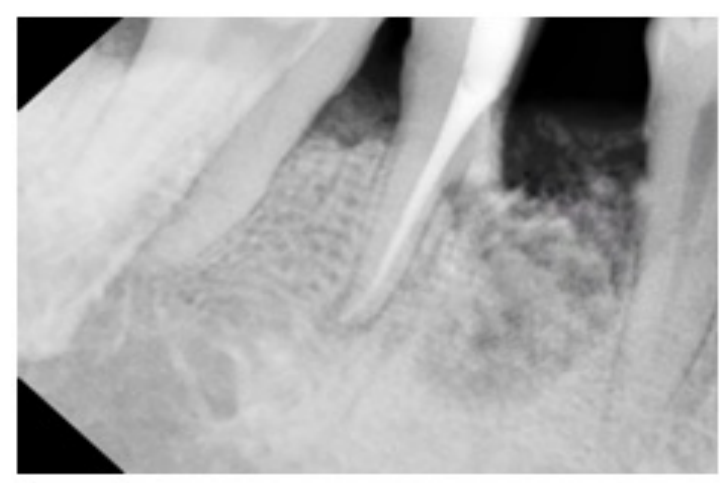

Figure 4b. 6 months post-operative radiograph

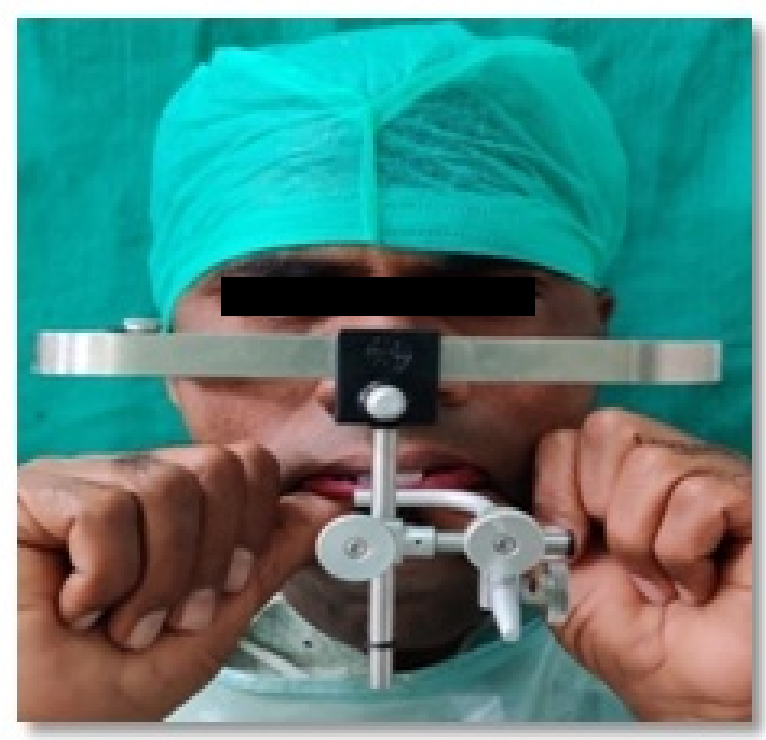

Figure 5a. Facebow record

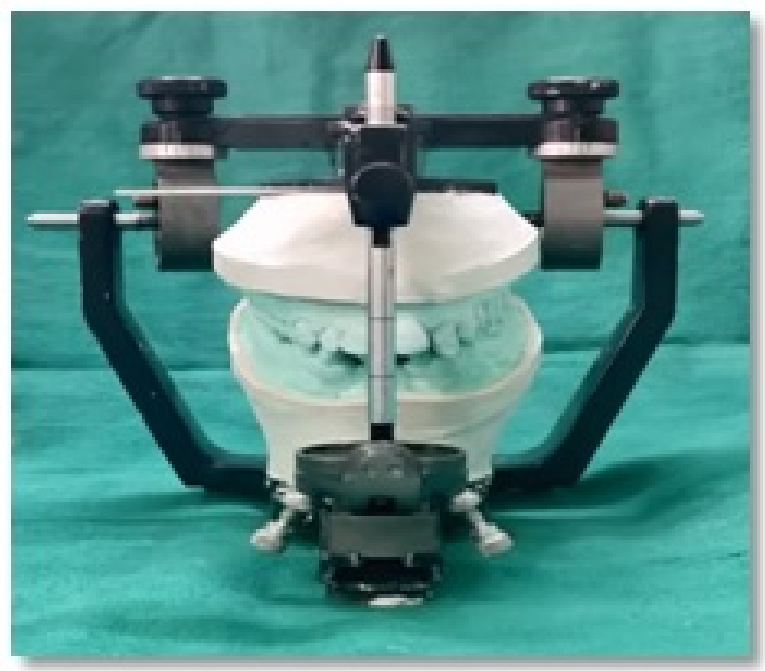

Figure 5b. Diagnostic mounting 


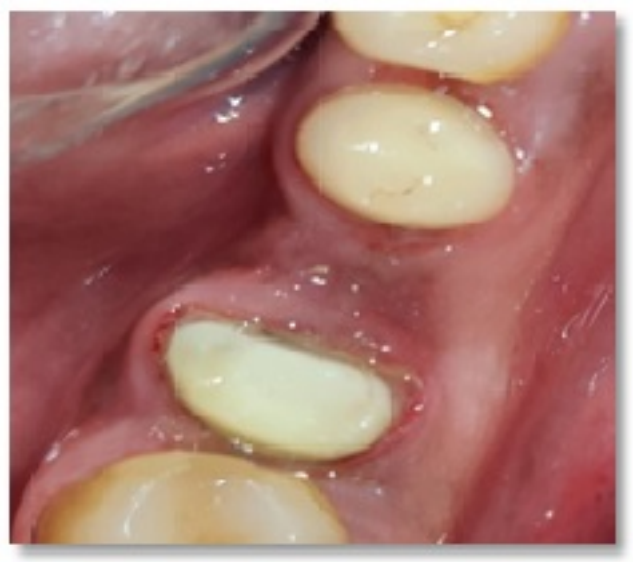

Figure 5c. Tooth preparation for prosthetic rehabilitation

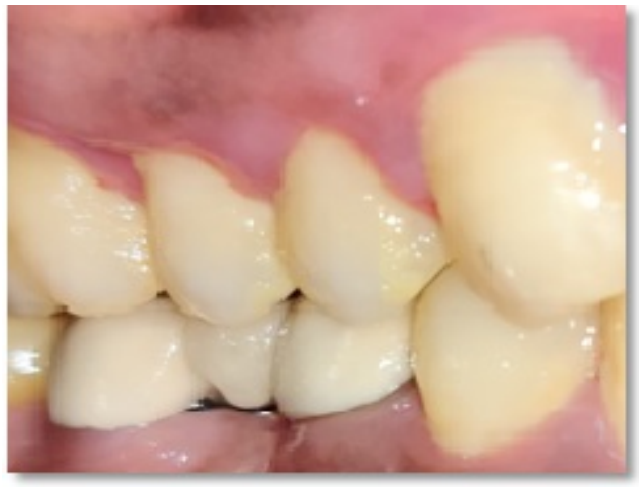

Figure 5d. Post - cementation lateral view

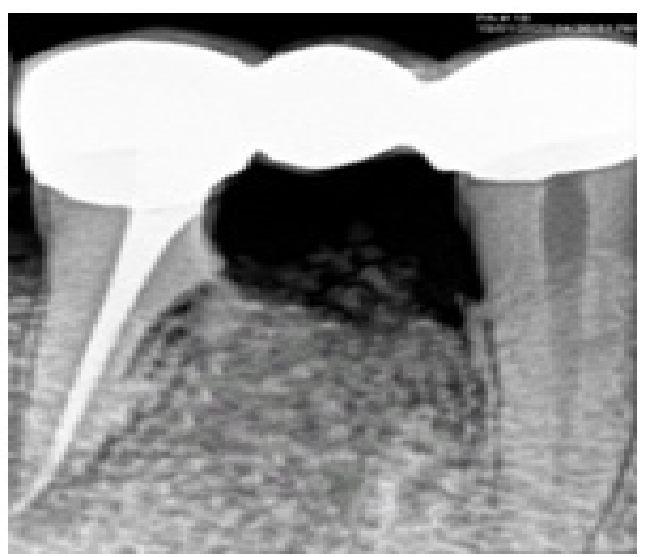

Figure 6. Post - cementation RVG at six months

\section{Discussion}

Hard and soft tissue regeneration, including the formation of new attachment housing, serves as the main stay of regenerative therapy. The treatment of a periodontal-endodontic defect includes debridement of root canals, as well as surgical approaches that give better access to clean the root surfaces and apical lesions as an adjunct. Bone loss due to pulpal disease is reversible, whereas advanced bone loss caused by periodontal disease is usually irreversible. [4] Multirooted, periodontally involved molars can be maintained for long periods of time with hemisection depending on their extent of bone destruction and bone present. This case was a perfect candidate for hemisection because the periodontal bone loss was more than $50 \%$ around mesial root which was less likely to resolve after non-surgical therapy alone [5]. The hemisection is a technique sensitive procedure. [1] Many factors determine the clinician's decision for choosing one treatment plan over another when confronted with a furcation invasion of a mandibular molar [6] e.g - Local factors like tooth anatomy, tooth mobility, crown root ratio, severity of attachment loss, inter-arch and intra-arch occlusal relationship, strategic dental value of tooth, general health of the patient and cost factors.

Tooth resection is indicated where there is severe vertical bone loss involving only one root of a multi rooted tooth, through and through furcation destruction, proximity of roots of adjacent teeth preventing adequate maintenance of oral hygiene, root exposure due to dehiscence, periodontal failure of an abutment tooth in a fixed bridge, if one root cannot be completely instrumented due to anatomic reasons or due to iatrogenic causes, vertical fracture of one root and/ or severe destruction of one root due to resorption, caries, trauma or perforation. Objectives of hemisection are to facilitate maintenance, prevent further attachment loss and obliterate furcation defects as a periodontal maintenance problem.

Park SY [7] performed root resection therapy on 691 molars in 579 patients. The associated factors were examined from 342 of 402 molars that had been followed up for more than one year and concluded that root resection to treat periodontal problems had a better prognosis than that for non-periodontal problems. To achieve a good result, it was important that the remaining roots had $>50 \%$ bone support. This ground rule may help to improve the predictability of root-resection therapy.

In a case report presented by Jain et al. [3] on a molar with excessive destruction of the mesial root due to the external root resorption and fair amount of the distal root remaining with adequate bone support, hemisection was carried out with the removal of the mesial root and crown. Remaining tooth structure was restored with composite and used as an abutment in crown and bridge after repositioning the occlusal contacts in favourable position, where they pointed out the success of hemisection as a viable treatment option. In the present case, the case selection criteria for performing a hemisection was optimum as the roots were not closely approximated or fused and surviving root was structurally capable of supporting a dowel and core restoration. Appropriate endodontic therapy was carried out prior to hemisection to avoid intrapulpal dystrophic calcification and postoperative tooth sensitivity. The case demonstrates successful management of tooth with endo-perio lesion and grade III furcation involvement by hemisectioning the mesial half of the tooth.

Hemisection has been used successfully to retain teeth with furcation involvement. However, there are few disadvantages associated with it. As with any surgical procedure, it can cause pain and anxiety. Root surfaces that are reshaped by grinding in the furcation or at the site of hemisection are more prone to caries. Often a favorable result may be cancelled out by decay after treatment. Failures of endodontic therapy due to any reason will washout the success of the treatment procedure. In addition, when the tooth has lost part of its root support, it will require a prosthetic rehabilitation to permit it to function independently or to serve as an abutment for a splint or bridge. Unfortunately, a restoration can contribute to periodontal destruction if the margins are defective or if non-occlusal surfaces do not have proper physiologic form. Also, an improperly shaped occlusal contact area may convert balanced forces into destructive forces and predispose the tooth to trauma from occlusion and ultimate failure 
of hemisection. [8] Bhuler (1988) [9] observed 32\% failure rate in hemisection case due to endodontic pathology and root fracture while long-term studies have shown greater success. In the present case, six months clinical, radiographic and functional follow-up showed good prognosis with the absence of mobility and healthy periodontal condition.

\section{Conclusion}

Hemisection may be considered as an alternative, effective and conservative treatment modality over conventional procedure or extraction of periodontally and endodontically affected teeth.

\section{References}

1. Akki S, Mahoorkar S (2011) Tooth hemisection and restoration an alternative to extraction - A case report. Int J Dent Clin 3: 67-68.

2. Radke U, Kubde R, Paldiwal A (2012) Hemisection: A window of hope for freezing tooth. Case Rep Dent 390874.
3. Jain A, Bahuguna R, Agarwal V (2011) Hemisection as an alternative treatment for resorbed multirooted tooth - A case report. Asian J Oral Hlth Allied Sci 1: 44-46.

4. Verma PK, Srivastava R, Gupta KK, Srivastava A (2011) Combined endodonticperiodontal lesion: A clinical dilemma. J Interdiscip Dent 1: 119-124.

5. Joshipura V (2011) Hemisection-A relevant, practical and successful treatment option. $J$ Int Oral Health 3: 43-48.

6. Napte B, Raghavendra SS (2014) Management of periodontally compromised mandibular molar with Hemisectioning: A case report. J Int Clin Dent Res Organ 6: 130-133.

7. Park SY, Shin SY, Yang SM, Kye SB (2009) Factors influencing the outcome of rootresection therapy in molars: A 10-year retrospective study. J Periodontol 80: 32-40. [Crossref]

8. Parmar G, Vashi P (2003) Hemisection : A Case Report and Review. Endodontology 15: 26-29.

9. Buhler H (1988) Evaluation of root resected teeth. Results after ten years. J Periodontol 59: 805-810. [Crossref]

Copyright: (2020 Parwani S. This is an open-access article distributed under the terms of the Creative Commons Attribution License, which permits unrestricted use, distribution, and reproduction in any medium, provided the original author and source are credited. 